\title{
PTS and Companding Transform for PAPR Reduction in OFDM
}

\author{
Roopam Pawar \\ Department of Electronics and Communication \\ Engineering \\ JUET, Guna \\ Madhya Pradesh, India
}

\author{
Abhishek Sharma \\ Department of Electronics and Communication \\ Engineering \\ JUET, Guna \\ Madhya Pradesh, India
}

\begin{abstract}
A combined model of Partial Transmit Sequence (PTS) and a Companding Transform is proposed to reduce Peak-toAverage Power Ratio (PAPR) in Orthogonal Frequency Division Multiplexing (OFDM). Simulation results demonstrate that the proposed scheme can substantially offer better PAPR reduction and BER performance.
\end{abstract}

\section{Keywords}

Complementary Cumulative Distribution Function (CCDF), Nonlinear Companding Transform (NCT), Orthogonal Frequency Division Multiplexing (OFDM), Partial Transmit Sequence (PTS), Peak-to-Average Power Ratio (PAPR).

\section{INTRODUCTION}

ORthogonal frequency division multiplexing (OFDM) has been attracting substantial attention due to its excellent performance under severe channel condition [1]. The rapidly growing application of OFDM includes WiMAX, DAB/DVB, and $4 \mathrm{G}$ wireless systems. However, one critical problem in OFDM is its high peak-to-average power ratio (PAPR) [1]. High PAPR increases the complexity of analog-to-digital and digital-to-analog converters, and lowers the efficiency of power amplifiers. Over the past decade various PAPR reduction techniques have been proposed [2]. Among all these techniques the simplest solution is to clip the transmitted signal when its amplitude exceeds a desired threshold. Clipping is a highly nonlinear process it produces significant out-of-band interference (OBI).

A good remedy for the OBI is the so-called companding. The companding transform may be the most significant and attractive method due to its effectiveness and simplicity. It can be employed straightforwardly without any restriction on the OFDM system parameters, i.e. the number of subcarriers, frame format and constellation type, but at the cost of the limited in-band distortion and out-of-band interference. The method was first proposed in [3], which employed the classical $\mu$-law transform and showed to be rather effective. Nevertheless, its average power of output signals is increased after such compression. Since then many different companding transforms with better performances have been published [4]-[7].

In order to achieve more efficient reduction in PAPR and improved BER perfomance, the paper introduced a new model using combination of two different PAPR reduction techniques one is among one of the very popular distortionless PAPR reduction technique i.e. Partial Transmit Sequence (PTS) which effectively reduces PAPR by selecting least peak power sample among all the scrambled samples [8]-[9]. Second is is a Nonlinear Companding Transform (NCT) which uses a smooth function, namely the airy special function [10].

The new proposed model exhibits advantages of both the techniques PTS which is distortionless and NCT, so improving overall system performance better than other PAPR reduction techniques.

The paper is organized as follows. In Section 2 the PAPR problem in OFDM is formulated briefly. Section 3 presents the proposed model comprises of PTS and NCT. Section 4 discusses the simulation performance. Section 5 draws the conclusion.

\section{PAPR IN OFDM}

Let $X(0), X(1), \ldots \ldots, X(N-1)$ represent the data sequence to be transmitted in an OFDM symbols with $\mathrm{N}$ subcarriers. The baseband representation of the OFDM symbol is given by (1)

$$
x(t)=\frac{1}{\sqrt{N}} \sum_{n=0}^{N-1} X(n) \exp \frac{j 2 \pi n t}{N} \quad 0 \leq t \leq T
$$

Where $T$ is the duration if the OFDM symbol. According to the central limit theorem, when $N$ is large, both the real and imaginary parts of $x(t)$ become Gaussian distributed, each with zero mean and variance of $\mathrm{E}\left[|x(t)|^{2}\right] / 2$, and the amplitude of the OFDM symbol follows a Rayleigh distribution. Consequently it is possible that the maximum amplitude of OFDM signal may well exceed its average amplitude. Practical hardware (e.g. A/D and D/A converters, power amplifiers) has finite dynamic range; therefore the peak amplitude of OFDM signal must be limited.

PAPR is mathematically defined as:

$$
P A P R=10 \log _{10} \frac{\max \left\lfloor\left. x(t)\right|^{2}\right\rfloor}{\frac{1}{T} \int_{0}^{T}|x(t)|^{2} d t}
$$

It is easy to see from (2) that PAPR reduction may be achieved by decreasing the numerator $\max \left[\left|x(t)^{2}\right|\right]$, increasing the denominator $(1 / T), \int_{0}^{T}|x(t)|^{2} d t$, or both.

The effectiveness of a PAPR reduction technique is measured by the Complementary Cumulative Distribution Function (CCDF), which is the probability that PAPR exceeds some threshold, i.e.

$$
\mathrm{CCDF}=\text { Probability }\left(\mathrm{PAPR}>\mathrm{p}_{0}\right),
$$


where, $\mathrm{p}_{0}$ is the threshold.

\section{PROPOSED MODEL}

In the proposed model as shown in Fig. 1, the IFFT output is sent to PTS block where the input data block is partitioned into disjoint subblocks or clusters which are combined to minimize the PAPR as shown in Fig. 2.

First, we define the data block as a vector, $X=\left[X_{0} X_{I} \ldots\right.$. $\left.X_{N-1}\right]^{T}$. Then, partition $X$ into $M$ disjoint sets, represented by the vectors $\left\{X_{m}, m=1,2, \ldots, M\right\}$. Here, we assume that the clusters consist of a contiguous set of subcarriers and are of equal size. The objective is to optimally combine the $M$ clusters

$$
X^{\prime}=\sum_{m=1}^{M} b_{m} X_{m}
$$

Where $\left\{b_{m}, m=1,2, \ldots, M\right\}$ are weighting factors $W$ and are assumed to be pure rotations. In the time domain

$$
x^{\prime}=\sum_{m=1}^{M} b_{m} x_{m}
$$

Where $x_{m}$, the IFFT of $X_{m}$, is called the partial transmit sequence. The phase factors are chosen to minimize the PAPR of $x$ '. Each sample is multiplies with the phase factor then find the sample which having least PAPR depends on the optimal phase factor.

Now the selected sample having least PAPR is further sent to NCT block, where companding function given in [10] will compress the signal amplitude by setting all the parameters given in [10].

The companding function $f(x)$ of the transform is given in (6)

$$
f(x)=\beta \cdot \operatorname{sign}(x) \cdot[\operatorname{airy}(0)-\operatorname{airy}(\alpha \cdot|x|)]
$$

Where, airy(.) is the airy function of the first kind. $\alpha$ is the parameter that controls the degree of companding and finally PAPR. $\beta$ is the factor adjusting the average output power of the compander to the same level as the average input power as given in (7)

$$
\beta=\sqrt{\frac{E\left[|x|^{2}\right]}{E\left[\operatorname{airy}(0)-\left.\operatorname{airy}(\alpha|x|)\right|^{2}\right]}}
$$

Where, E[.] denotes the expectation.

The decompanding function is the inverse of $f(x)$ as given in (8)

$$
f^{-1}(x)=\frac{1}{\alpha} \cdot \operatorname{sign}(x) \cdot \operatorname{airy}^{-1}\left[\operatorname{airy}(0)-\frac{|x|}{\beta}\right]
$$

Notice that the input to the decompander is a quantized signal with finite set of values. We can therefore numerically precompute inverse of $f(x)$ and use table look-up to perform the decompanding in practice. In Fig. 3 Companding and decompanding profile is given. From simulation result in Section 4 , we can verify the PAPR reduction and improved BER performance.

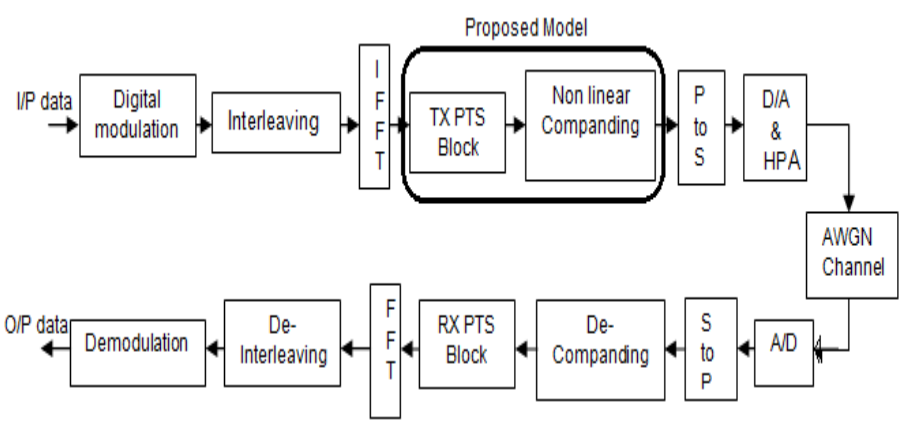

Fig. 1 Block diagram of OFDM system with proposed model

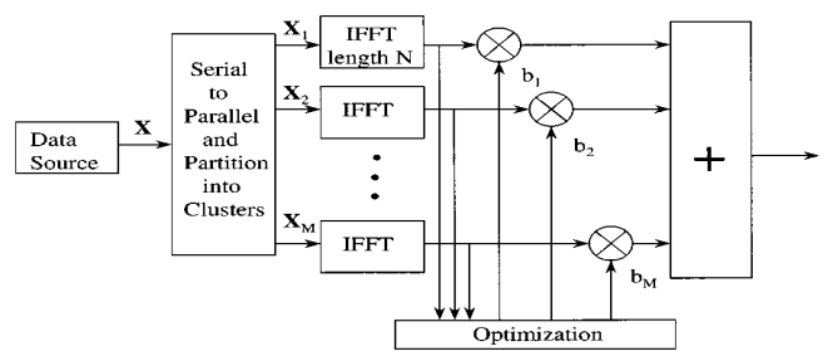

Fig.2 Block diagram of PTS approach
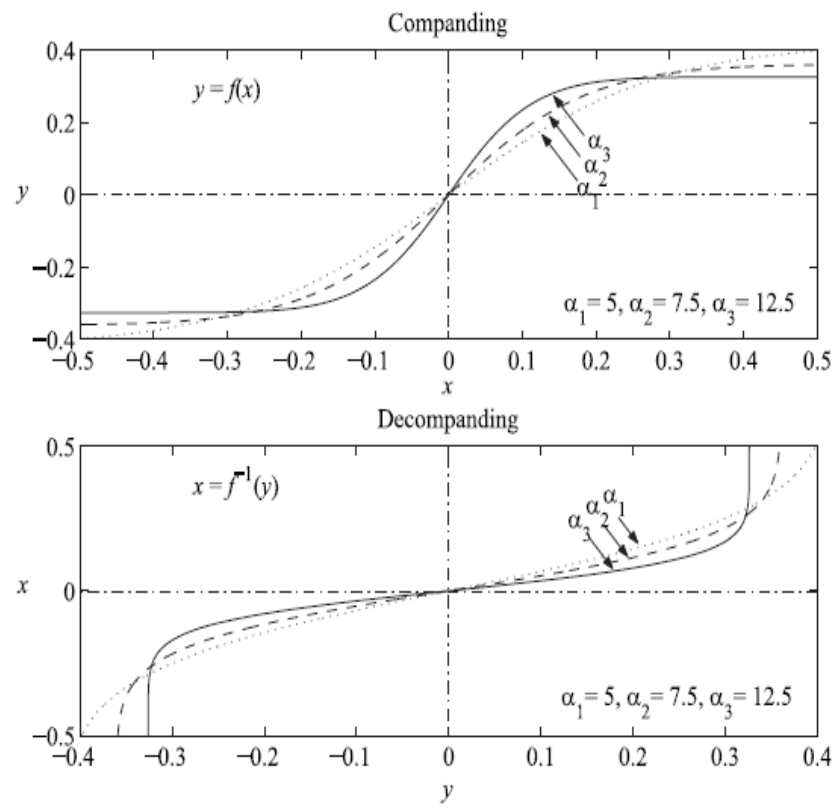

Fig.3 Companding and decompanding profile

\section{SIMULATION PERFORMANCE}

We consider an OFDM system with number of subcarriers $N$ $=256$, QPSK Modulation, number of sub-blocks $M=64$, over-sampling factor $l=4$, weighting factor $W=4$, the parameter $\alpha$ in the companding function is chosen to be 30 . Consecutively about 19.6 percent of companded signal is within the noise-suppression range of the decompanding function and compander input power is $3 \mathrm{dBm}$. It is assumed that symbol timing is perfect and the frequency offset is absent at the receiver.

As shown in Fig.4, the proposed model can significantly reduce PAPR. The PAPR of the proposed model is approximately $2.9 \mathrm{~dB}$ which is $10.2 \mathrm{~dB}, 3 \mathrm{~dB}$ and $1 \mathrm{~dB}$ better 
than original signal, $\mu$-law and NCT techniques respectively at $\mathrm{CCDF}=10^{-3}$. The comparison of CCDF of PAPR of proposed model with various companding schemes is given in Table 1.

Fig. 5 shows that the proposed scheme provides better BER as compared to other companding schemes. The $\mathrm{E}_{\mathrm{b}} / \mathrm{N}_{0}$ of proposed scheme is approximately $11.2 \mathrm{~dB}$ which is $3 \mathrm{~dB}$ higher than original signal and $2.8 \mathrm{~dB}$ better than $\mu$-law companding scheme at $\mathrm{BER}=10^{-4}$. Comparison of $\mathrm{BER}$ performance of various companding scheme with proposed model is given in Table 2 .

Table 1. Comparison of PAPR of proposed model with various companding schemes

\begin{tabular}{|c|c|}
\hline COMPANDING SCHEMES & PAPR $(\mathbf{d B})$ at $\mathbf{C C D F}=\mathbf{1 0}^{\mathbf{3}}$ \\
\hline Original Signal & 13.1 \\
\hline$\mu$-law in [11] & 5.9 \\
\hline NCT in [10] & \\
\hline Proposed (PTS+NCT) & 3.9 \\
\hline
\end{tabular}

Table 2. Comparison of BER performance of proposed model with various companding schemes

\begin{tabular}{|c|c|}
\hline COMPANDING SCHEMES & $\mathbf{E}_{\mathbf{b}} / \mathbf{N}_{\mathbf{0}}$ at BER $=\mathbf{1 0}^{\mathbf{4}}$ \\
\hline Original Signal & 8.2 \\
\hline$\mu-$-law in [11] & $\sim 14$ \\
\hline Proposed (PTS+NCT) & 11.2 \\
\hline
\end{tabular}

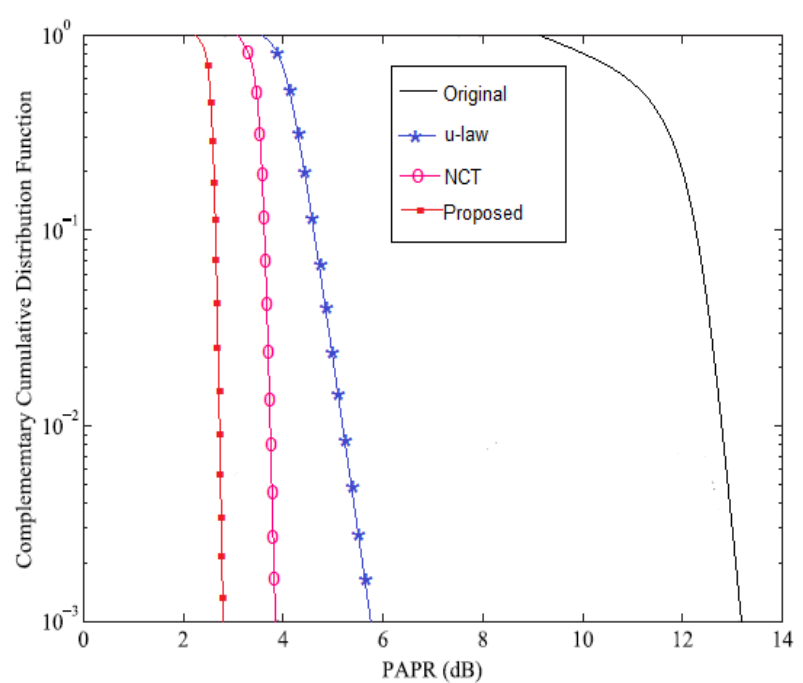

Fig. 4 CCDF statistics of comparison of proposed model (PTS+NCT) with various Companding schemes and original signal

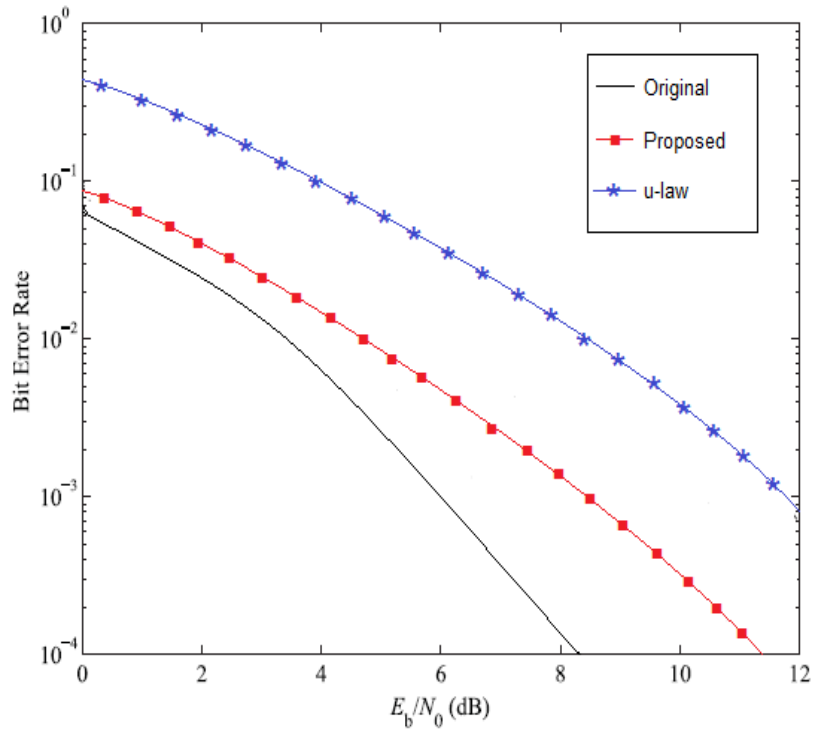

Fig. 5 BER versus $E_{b} / N_{0}$ comparison of proposed model (PTS+NCT) with $\mu$-law companding and original signal

\section{CONCLUSION}

A combined model of two different PAPR reduction techniques i.e. Partial Transmit Sequence (PTS) and Nonlinear Companding Transform (NCT) is proposed and simulated. The model reduces PAPR better than other techniques and improves BER performance as shown in Fig. 4 and Fig. 5 respectively. Depends on the value of $\alpha$ for NCT and the value of $M$ for PTS, overall syatem performance can be improved remarkbly. Thus, this is in line with the preceding analyses. The proposed model can be used where the overall system performance is essential along with the PAPR reduction.

\section{ACKNOWLEDGMENTS}

The authors would like to thank the anonymous reviewers for their valuable comments and suggestions.

\section{REFERENCES}

[1] R. van Nee and R. Prasad, OFDM for Wireless Multimedia Communications. Boston, MA: Artech House, 2000.

[2] S. H. Han and J. H. Lee, "An Overview of peak-toaverage power ratio reduction techniques for multicarrier transmission," IEEE Wireless Commun., vol. 12, pp. 5665, Apr. 2005.

[3] X. Wang, T. T. Tjhung, and C. S. Ng, "Reduction of peak-to-average power ratio of OFDM system using a companding technique," IEEE Trans. Broadcast., vol. 45, no. 3, pp. 303-307, Sept. 1999.

[4] T. Jiang and G. Zhu, "Nonlinear companding transform for reducing peak-to-average power ratio of OFDM signals," IEEE Trans. Broadcast., vol. 50, no. 3, pp. 342346, Sept. 2004.

[5] T. Jiang, Y. Yang, and Y. Song, "Exponential companding technique for PAPR reduction in OFDM systems," IEEE Trans. Broadcast., vol. 51, no. 2, pp. 244-248, June 2005. 
[6] D. Lowe and X. Huang, "Optimal adaptive hyperbolic companding for OFDM," in Proc. IEEE Second Intl Conf. Wireless Broadband and Ultra Wideband Commun., pp. 24-29, Aug. 2004.

[7] T. Jiang and Y. Wu, "An overview: peak-to-average power ratio reductio techniques for OFDM signals," IEEE Trans. Broadcast., vol. 54, no. 2, pp. 257-268, June 2008.

[8] L.J. Cimini, N.R. Sollenberger, "Peak-to-average power reduction of an OFDM signal using Partial Transmit Sequences," IEEE Commun. Lett., vol. 4, no. 3, Mar. 2000.
[9] S. H. Muller and J. B. Hüber, "OFDM with reduced peak-to-average power ratio by optimum combination of partial transmit sequences,"Electron. Lett., vol. 33, no. 5, pp. 368-369, Feb. 1997.

[10] Y. Jiang, "New companding transform for PAPR reduction in OFDM", IEEE Comm. Lett. vol.14, no. 4, Apr 2010.

[11] X. B.Wang, T. T. Tjhung, and C. S. Ng, "Reduction of peak-to- average power ratio of OFDM system using a companding technique," IEEE Trans. Broadcast., vol. 45, no. 3, pp. 303-307, Sep. 1999. 